\title{
TESTS OF RANDOM DENSITY MODELS OF TERRESTRIAL PLANETS
}

\author{
William M. Kaula \\ Department of Earth \& Space Sciences \\ University of California, Los Angeles \\ Paul D. Asimow \\ Department of Earth \& Planetary Sciences \\ Harvard University, Cambridge
}

\begin{abstract}
Random density models are analyzed to determine the low degree harmonics of the gravity field of a planet, and therefrom two properties: an axiality $\mathbf{P}_{l}$, the percent of the degree variance in the zonal term referred to an axis through the maximum for degree $l$; and an angularity $\mathrm{E}_{l n}$, the angle between the maxima for two degrees $l, n$. The random density distributions give solutions reasonably consistent with the axialities and angularities for the low degrees, $l<5$, of Earth, Venus, and Moon, but not for Mars, which has improbably large axialities and small angularities. Hence the random density model is an unreliable predictor for the nonhydrostatic second-degree gravity of Mars, and thus for the moment-of-inertia, which is more plausibly close to $0.365 \mathrm{MR}^{2}$.
\end{abstract}

\section{Introduction}

Bills (1989) argued that the moment-of-inertia of Mars should be close to $0.345 \mathrm{MR}^{2}$, where $\mathrm{M}$ is the planet mass and $\mathbf{R}$ is the radius. This was appreciably lower than the $0.365 \mathrm{MR}^{2}$ favored by Reasenberg (1977) and Kaula (1979), and has considerable significance for stress levels and composition in Mars (Kaula et al., 1989).

The basis for the argument of Bills (1989) was that the equality of the equatorial non-hydrostatic momentsof-inertia, $\delta \mathrm{C}=\delta \mathrm{B}$, implicit in the higher value 0.365 $\mathrm{MR}^{2}$, is extremely improbable for random density distributions in a planet. The lower value of $0.345 \mathrm{MR}^{2}$ corresponds to the most probable condition of $\delta C-\delta \mathrm{B}=$ $\delta$ B $-\delta$ A. Earth, Venus, and Moon all are close to this condition, as indicated by figure 1, which is a histogram of the quantity

$$
f=(\delta \mathrm{C}-\delta \mathrm{B}) /(\delta \mathrm{C}-\delta \mathrm{A})
$$

Figure 1 was generated from 30,000 configurations of 30 masses random in magnitude and location on a sphere. It is essentially identical with figure 2 of Goldreich \& Toomre (1969) and figure 2 of Bills (1989). R-Mars on figure 1 corresponds to the $\delta C=6 \mathrm{~B}$ assumed by Reasenberg (1977) and Kaula (1979), while B-Mars corresponds to the $\delta B=(\delta C+\delta A) / 2$ of Bills (1989).

In calculating figure 1 and all subsequent figures it is assumed that the 3-axis coincides with the minimum of the second-degree component of the non-hydrostatic gravity field, because any plausible terrestrial planet has sufficient dissipation to cause a drift to rotation about the axis of maximum moment-of-inertia on a time scale small

Copyright 1991 by the American Geophysical Union.

Paper number $91 \mathrm{GL} 01064$

0094-8534/91/91GL 01064\$03.00

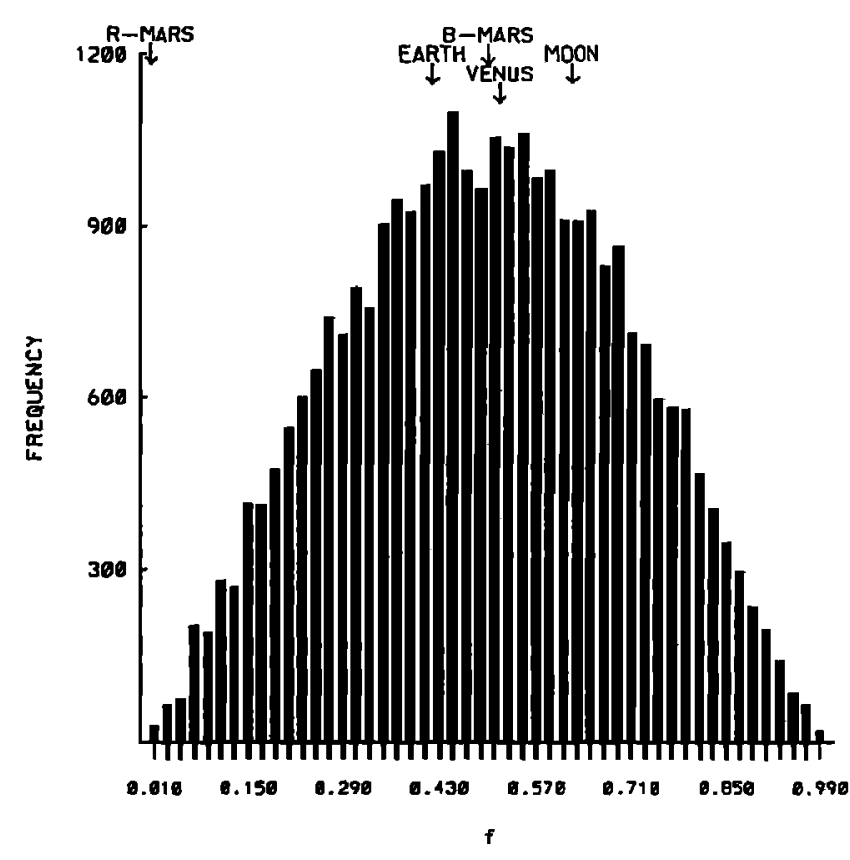

Fig. 1. Probability density distribution of the difference ratio $f=(\mathrm{C}-\mathrm{B}) /(\mathrm{C}-\mathrm{A})$. All of figures 1-7 are from 30,000 random configurations, each containing 30 miasses random in mignitude and location on a sphere.

compared to that of the generation of density irregularities (Goldreich and Toomre, 1969). It is then convenient to place the 1 -axis through the maximum of the second-degree component.

If a property predicted by a physical model (such as a random density distribution on a sphere) is not observable, then it is desirable to seek other properties that are observable to test the model. Neither the precession nor the non-hydrostatic $C_{20}$ of Mars, alternatives to infer its $\mathrm{I} / \mathrm{MR}^{2}$, are presently observable. But there are other properties of Mars's gravity field which are both predicted by a random density distribution and observed. If the predictions do not give a reasonable probability for the observed values, then the model is likely to be inapplicable for the non-observed properties.

\section{Analysis}

Properties of Mars's gravity field that are both predictable and observable are, for any spherical harmonic degree $l>2$ :

1. The percent $\mathrm{P}_{l}$ of the variance for degree $l$ that is in the zonal term for a coordinate system that has its 
polar axis through the maximum. We call this property the axiality.

And for any pair of spherical harmonic degrees $l, n$ :

2. The angle $E_{l n}$ between the maximum for degree $l$ alone and the maximum for degree $n$ alone. We call this property the angularity.

If Mars's gravity field is dominated by a single feature that is near to axial symmetry, such as Tharsis, then for $l$, $n$ small we should expect the angles $\mathrm{E}_{\boldsymbol{l n}}$ to be improbably small according to the random density model, and the percents $P_{l}$ to be improbably large.

To obtain the axiality $\mathbf{P}_{l}$, we use the fact that the maximum must be represented solely by the zonal term of spherical harmonics referred to an axis through the maximum:

$$
\mathrm{P}_{l}=\mathrm{M}_{l}^{2} /\left[(2 l+1) \sigma l^{2}\right]
$$

where $\mathrm{M}_{l}$ is the maximum value for degree $l$, located at $\theta_{l}, \lambda_{l}, \quad 2 l+1$ is the square of the normalized zonal harmonic at the pole, and $\sigma l^{2}$ is the degree variance, the sum of the squares of the normalized coefficients. The maximum $\mathrm{M}_{I}$ is the sum of coefficients times harmonics at its location $\theta_{l}, \lambda$;

$$
\underset{\sin m \lambda l}{\mathrm{M}_{l}=\Sigma_{m}}\left(\begin{array}{l}
\mathrm{C}_{l m}\left(\cos m{ }_{l m}\right. \\
\left.\cos \theta_{l}\right),
\end{array}\right.
$$

where $P_{l m}$ is the associated Legendre polynomial. If the axes are rotated so that the 3-axis is through the maximum, then the only non-zero term at $\theta_{l}, \lambda l$ is the zonal, $\mathrm{m}=0$. The degree variance $\sigma l^{2}$ is the sum of the squares of the coefficients,

$$
\sigma l^{2}=\Sigma_{m}\left(\mathrm{C}_{l m^{2}}+\mathrm{S}_{l m^{2}}\right),
$$

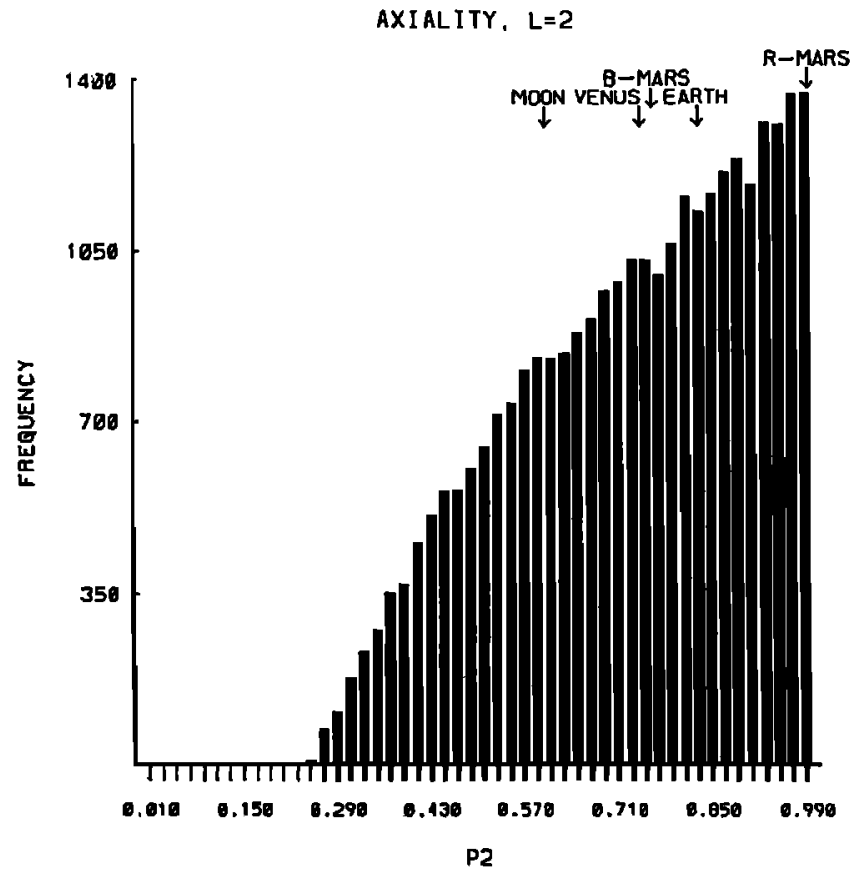

Fig. 2. Probability density distribution of the seconddegree axiality $P_{2}$, defined by eq. (2), with observed values for Earth, Venus, and Moon, and hypothetical values for Mars: R-Mars, consistent with Reasenberg (1977), and B-Mars, consistent with Bills (1989).
AXIALITY, L $=3$

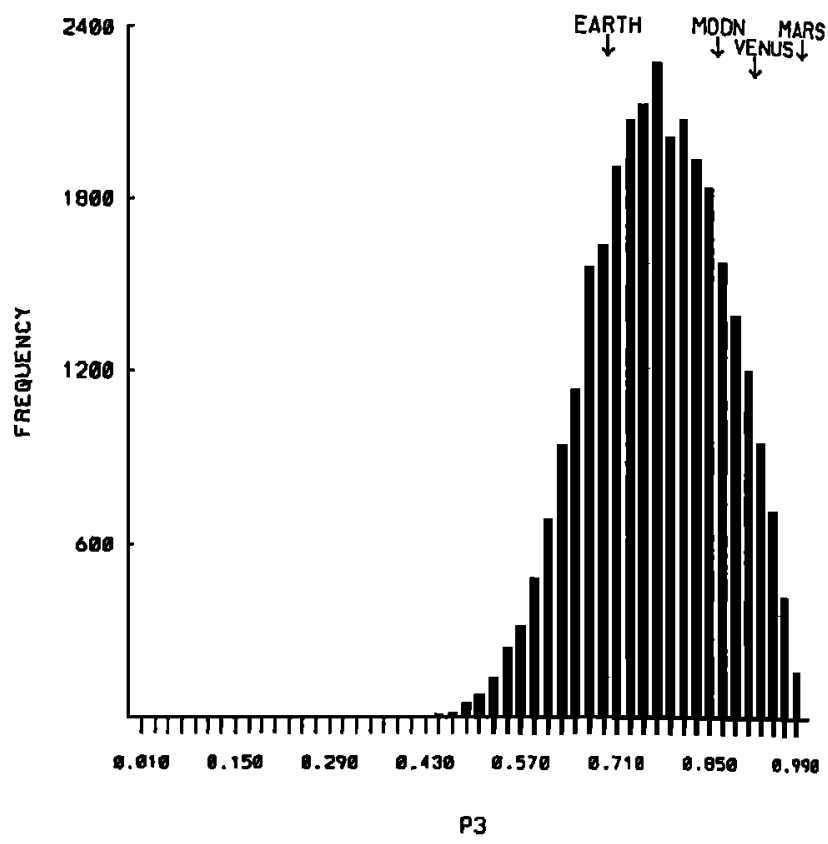

Fig. 3. Probability density distribution of the third degree axiality $\mathrm{Ps}_{\mathrm{s}}$, with observed values for all planets.

for spherical harmonics normalized so that their mean square is unity.

To obtain the angularity $E_{l n}$ between harmonic degrees $l$ and $n$, apply the cosine law to the maxima locations $\theta_{l}, \lambda_{l}$ and $\theta_{n}, \lambda_{n}$ :

$$
\begin{array}{r}
\cos \mathrm{E}_{l n}=\cos \theta_{l} \cos \theta_{n} \\
+\sin \theta_{l} \sin \theta_{n} \cos \left(\lambda_{l}-\lambda_{n}\right)
\end{array}
$$

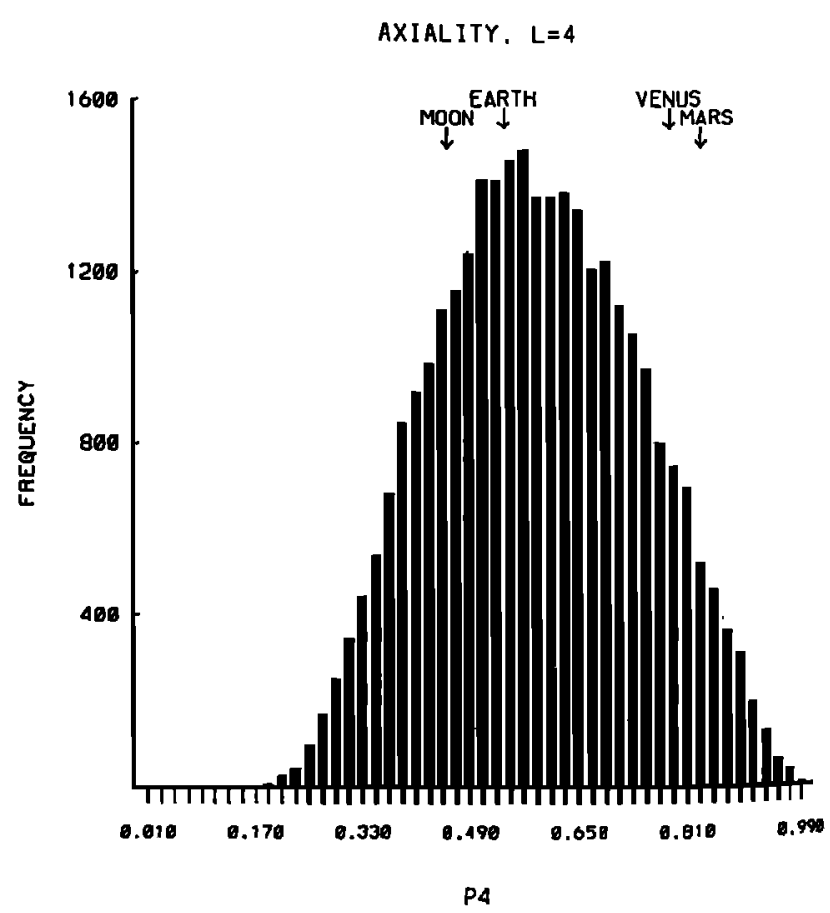

Fig. 4. Probability density distribution of the fourth degree axiality $\mathrm{P}_{4}$, with observed values for all planets. 
The results from 30,000 configurations of 30 random masses for axialities $P_{2}, P_{3}$, and $P_{4}$ are given in figures 2 through 4, and for angularities $E_{23}, E_{24}$, and $E_{34}$ in figures 5 through 7 .

The axiality $\mathrm{P}_{2}$ is related to the function $f$ shown in figure 1. For references axes that are principal axes, only $\mathrm{C}_{20}$ and $\mathrm{C}_{22}$ are non-zero; using the relationships thereof to the non-hydrostatic moments $\delta \mathrm{A}, \delta \mathrm{B}$, and $\delta \mathrm{C}$ (Bills, 1989; Kaula et al., 1989), and eqs. (1), (2) above:

$$
\mathbf{P}_{2}=\left(4-4 f+f^{2}\right) /\left(4-4 f+4 f^{2}\right)
$$

The bunching up of the $\mathbf{P}_{2}$ histogram toward unity is consistent with $\mathrm{dP}_{2} / \mathrm{d} f$ going to zero with $f$.

On the figures are also marked the values for the actual terrestrial planets. The gravity fields used were those of Christensen \& Balmino (1979), Bills \& Ferrari (1980), Bills et al. (1987), and Marsh et al. (1988).

\section{Conclusions}

The figures indicate that the random density model is not inconsistent with observation for Earth, Moon, and Venus, but quite inconsistent for Mars, which has improbably large $P_{3}$ and $P_{4}$, and improbably small $E_{2} s$, E24, and Es4. These probabilities are given in Table 1.

Because the random density model does not agree with observable properties of Mars, it is an unreliable predictor for the unobservable second-degree axiality $\mathrm{P}_{2}$ of Mars and thence the moment-of-inertia ratio I/MR ${ }^{2}$ connected thereto. The second-degree components of the gravity field are quite similar to the higher degree components in their couse: there is no special mechanism peculiar thereto, analogous to the Coriolis effect on the magnetic dipole. Where the second-degree components

ANGULARITY, L=2.3

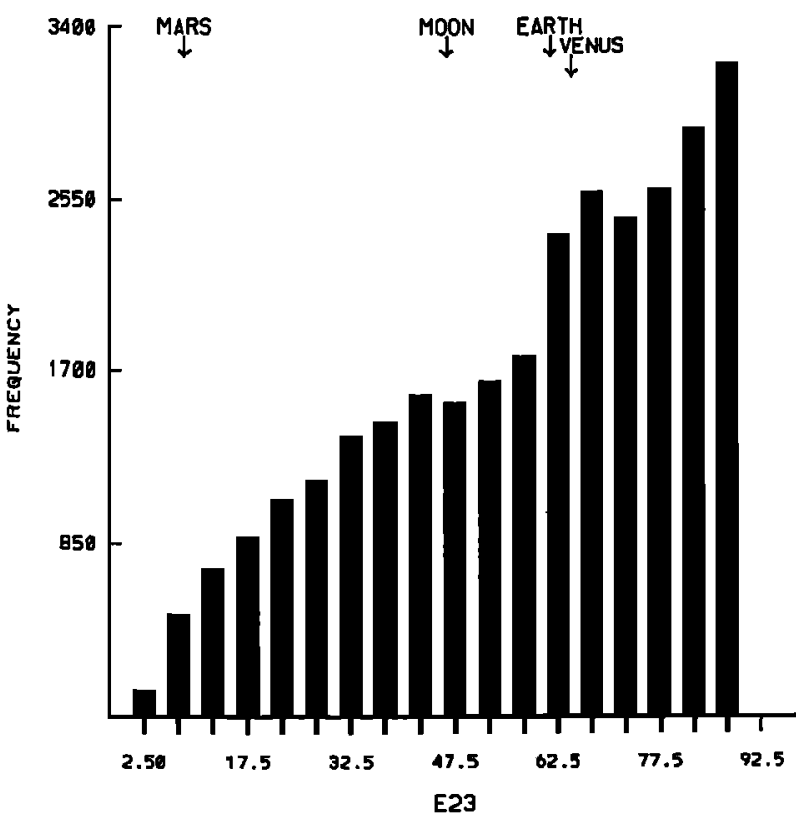

Fig. 5. Probability density distribution of the angularity between the second and third degrees $\mathrm{E}_{2} \mathrm{~s}$, defined by eq. (5), with observed values for all planets.
ANGULAFITY, L $=2.4$

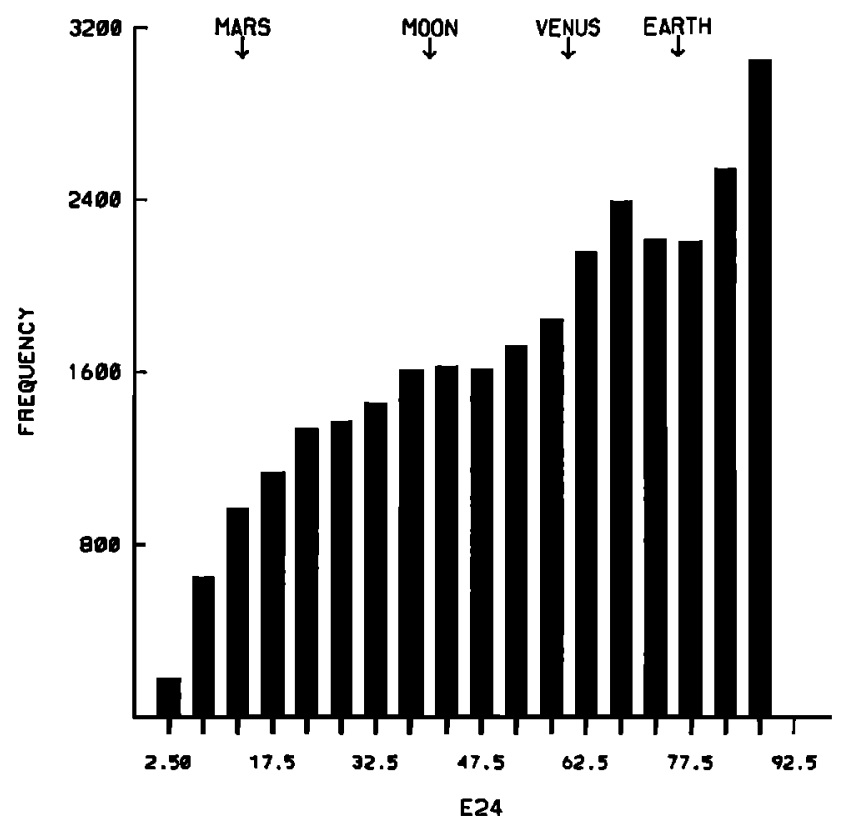

Fig. 6. Probability density distribution of the angularity between the second and fourth degrees $\mathrm{E}_{24}$, with observed values for all planets.

differ is in their effect, which is to determine the orientation and, to a minor degree, the rate of rotation. Given the similarity of cause, the high axialities of the third and fourth degree harmonics about axes close to the maximum of the second degree suggest a similar axiality

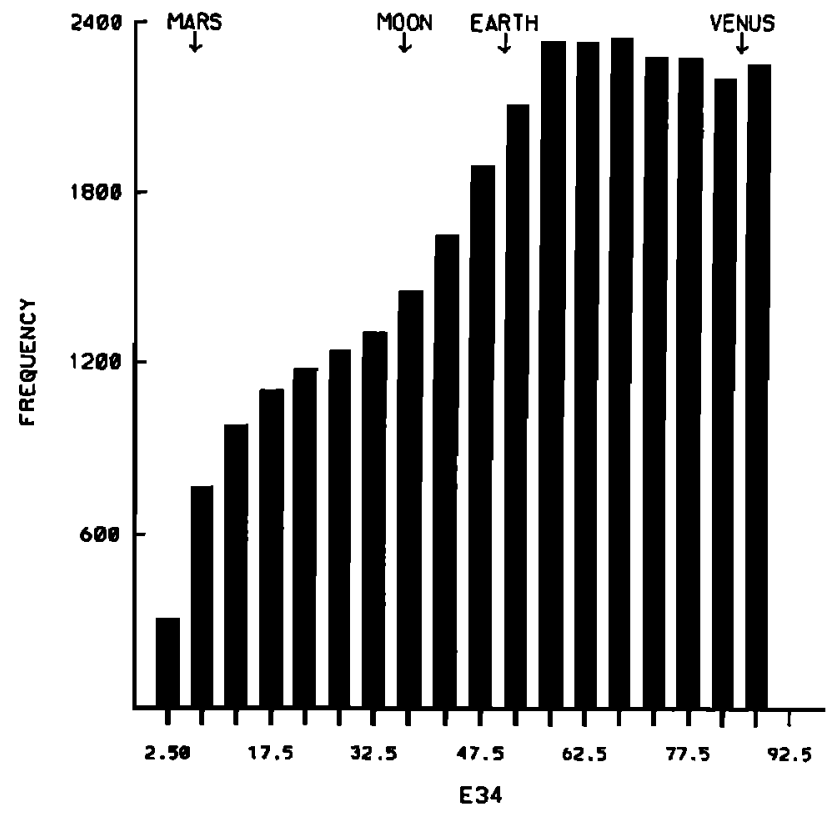

Fig. 7. Probability density distribution of the angularity between the third and fourth degrees Es 4 , with observed values for all planets. 
TABLE 1: Magnitudes and Probabilities of More Extreme Values of Observed Martian Properties

Property Symbol Magnitude Probability

\begin{tabular}{llrl} 
Third-Degree Axiality & Ps $_{3}$ & 0.987 & 0.004 \\
Fourth-Degree Axiality & $P_{4}$ & 0.827 & 0.063 \\
Second-Third Angularity & $\mathrm{E}_{23}$ & $10.78^{\mathrm{O}}$ & $\mathbf{0 . 0 1 3}$ \\
Second-Fourth Angularity & $\mathrm{E}_{24}$ & $14.84^{\mathrm{o}}$ & $\mathbf{0 . 0 4 7}$ \\
Third-Fourth Angularity & $\mathrm{E}_{34}$ & $\mathbf{8 . 4 2 ^ { \mathrm { O } }}$ & $\mathbf{0 . 0 1 4}$ \\
\hline
\end{tabular}

in the second degree, and hence a most probable value for $\mathbf{I} / \mathbf{M R}^{2}$ of 0.365 or slightly less.

Any attempt to predict the second-degree component of the gravity field entails, implicitly if not explicitly, a model to connect the degrees. The most obvious model is axially symmetric support for an axially symmetric load centered on Tharsis, such as a poloidal mantle convection or an elastic shell, as computed by Sleep and Phillips (1985). The simplest axially symmetric load-plusresponse is a point mass on the 1-axis. An attempt to fit such a point mass to the observed harmonics, $h m=2,2$ through 4,4, produced a rather poor result: this opposite extreme of a random mass distribution is about as poor. The load-plus-response must extend quite far from the axis.

The apparent consistency of random density distributions with the properties of the other terrestrial bodies probably arises from their being more complex tectonic systems, in which no one feature is dominant.

Acknowledgements. Paul D. Asimow was supported for a visit to U.C.L.A. by the NASA Planetary Geology and Geophysics Undergraduate Research Program.

\section{REFERENCES}

Bills, B. G., The moments of inertia of Mars, Geophys. Res. Let., 16, 385-389, 1989.

Bills, B. G. and A. J. Ferrari, A harmonic analysis of lunar gravity, J. Geophys. Res., 85, 1013-1025, 1980.

Bills, B. G., W.S. Kiefer, and R. L. Jones, Venus gravity: a harmonic analysis, J. Geophys. Res., 92, 10,335-10,351, 1987.

Christensen, E. J. and G. Balmino, Development and analysis of a twelfth degree and order gravity model for Mars, J. Geophys. Res., 84, 7943-7953, 1979.

Goldreich, P. and A. Toomre, Some remarks on polar wandering, J. Geophys. Res., 74, 2555-2567, 1969.

Kaula, W. M., N. H. Sleep, and R. J. Phillips, More about the moment-of-inertia of Mars, J. Geophys. Res., 16, 1333-1336, 1989.

Marsh, J. G. and 19 others, A new gravitational model for the Earth from satellite tracking data: GEM-T1, J. Geophys. Res., 93, 6169-6215, 1988.

Reasenberg, R. D., The moment of inertia and isostasy of Mars, J. Geophys. Res., 82, 369-375, 1977.

Sleep, N.H., and R. J. Phillips, Gravity and lithospheric stress on the terrestrial planets with reference to the Tharsis region of Mars, J. Geophys. Res., 80, 4469$4489,1985$.

William M. Kaula

Department of Earth \& Space Sciences

University of California, Los Angeles

Los Angeles, CA 90024-1567

Paul D. Asimow

Department of Earth \& Planetary Sciences

Harvard University

Cambridge, MA 02138

(Received July 25, 1990;

Revised October 18, 1990

Accepted October 20, 1990) 